\title{
A novel CCM1 mutation associated with multiple cerebral and vertebral cavernous malformations
}

\author{
Silvia Lanfranconi ${ }^{1}$, Dario Ronchi ${ }^{2}$, Naghia Ahmed ${ }^{2}$, Vittorio Civelli ${ }^{3}$, Paola Basilico ${ }^{1,2}$, Nereo Bresolin ${ }^{1,2}$, \\ Giacomo Pietro Comi ${ }^{1,2}$ and Stefania Corti ${ }^{1,2^{*}}$
}

\begin{abstract}
Background: Cerebral cavernous malformations are relatively rare vascular disorders that may affect any part of the central nervous system. This presentation has been associated with heterozygous mutations in CCM1/KRIT1, CCM2/ malcavernin and CCM3/PDCD10. We aimed to investigate the genetic defect underlying multiple cerebral and vertebral cavernous malformations in a multigenerational Italian family.

Case presentation: The proband is a 49-year-old man who underwent cerebral MRI in his thirties for persistent haeadache and tingling in his left arm and leg and was diagnosed with multiple supratentorial cavernous angiomas. A right frontal angioma with radiological evidence of a recent bleeding was surgically removed when he was 39 years old and he was thereafter asymptomatic. Magnetic resonance imaging revealed multiple cerebral cavernous malformations in seven members of his familily. Four subjects were asymptomatic. Other family mambers displayed heterogeneous clinical features including seizures and recurrent brain haemorrhages. Sequence analysis in the proband disclosed a novel heterozygous nucleotide substitution (c.263-10A > G) in intron 5 of CCM1. This variant is predicted to create an abnormal acceptor splice site and segregated in affected relatives available for molecular screening. The analysis of CCM1 transcript in proband's lymphocytes confirmed the partial retention of intron 3 resulting in a premature termination codon.
\end{abstract}

Conclusions: Our findings demonstrate that c.263-10A > G mutation is associated with cerebral cavernous malformations. A better knowledge of the disease-associated phenotype may lead to an early diagnosis and to an appropriate clinical surveillance in affected patients.

Keywords: Cerebral Cavernous Malformations, CCM1 protein, Krev interaction trapped 1 protein, Hemangioma, Cavernous, Central Nervous System

\section{Background}

Cerebral cavernous malformations (CCM) are vascular defects consisting of clusters of enlarged, thin-walled and leaky capillaries without intervening nervous tissue. Estimated prevalence is $0.1-0.5 \%$ in general population [1,2].

The clinical phenotype is highly variable. CCMs mainly occur in the central nervous system (CNS) where they appear as focal lesions with a peripheral T2 hypointensity due to hemosiderin deposition which can be

\footnotetext{
* Correspondence: stefania.corti@unimi.it

'Neurology Unit, IRCCS Foundation Ca' Granda Ospedale Maggiore

Policlinico, Milan, Italy

${ }^{2}$ Dino Ferrari Center, Neuroscience Section, Department of Pathophysiology and Transplantation (DEPT), IRCCS Foundation Ca' Granda Ospedale Maggiore Policlinico, University of Milan, Via Francesco Sforza 35, 20122 Milan, Italy

Full list of author information is available at the end of the article
}

detected with higher sensitivity on T2* GRE sequences. The involvement of other districts has been so far poorly investigated. Indeed, CCMs can be clinically silent $(60 \%)$ or lead to recurrent headache, focal neurological deficits, cerebral haemorrhage (41\%) and seizures (45\%) [3].

Most of CCM cases are sporadic but familial forms, showing autosomal dominant pattern of inheritance, have been described [4].

Germline mutations in three genes account for $90 \%$ of familial cases: CCM1 (also known as KRIT1), CCM2 and PDCD10 (CCM3) [3]. The analysis of affected tissues has often disclosed a second somatic mutation, which is likely required to prime the pathogenetic cascade, according a two-hit mechanism [5].

CCM1 mutations were disclosed in more than $40 \%$ of familial cases but incomplete penetrance is not uncommon, 
being reported in $60-80 \%$ of mutated families. CCM1 encodes for the krev interaction trapped 1 (KRIT1) protein, which is involved in maintaining the integrity of endothelial junctions, and may play a role in microtubule targeting $[6,7]$.

Here we describe clinical, neuroradiological and molecular features of a novel Italian family with multiple cerebral and vertebral cavernous malformations harbouring a novel CCM1 splicing mutation.

\section{Case presentation}

\section{Clinical features}

We studied a four generations Italian pedigree in which several subjects showed multiple cerebral cavernous malformations (Figure 1).

The proband (III-1) is a 49-year-old man. At 39 years of age he complained headache and pins and needles in his left arm and leg. Cerebral MRI performed at that time revealed multiple cerebral supratentorial angiomas with radiological evidence of recent bleeding in the context of a right frontal lesion, which was surgically removed in 2004. Cerebral MRI findings were stable over time. Spinal MRI was negative for spinal lesions but revealed multiple vertebral hemangiomas whose size and number were unexpected for his age (Figure 2D-F). The patient still complains headache, but he is otherwise asymptomatic (last neurological examination performed in September 2013).

Patient's 46-year-old brother (III-2) experienced sudden onset of right facial numbness at 40 years of age. Cerebral MRI displayed a recent bleeding within a cavernous angioma close to the right cerebellar peduncle and a further lesion in the pons (GRE sequences not performed). Symptoms resolved and he was asymptomatic until August 2013 when he complained headache followed by right facial numbness, vertigo, nausea and gait instability. Cerebral CT revealed acute bleeding in the context of the

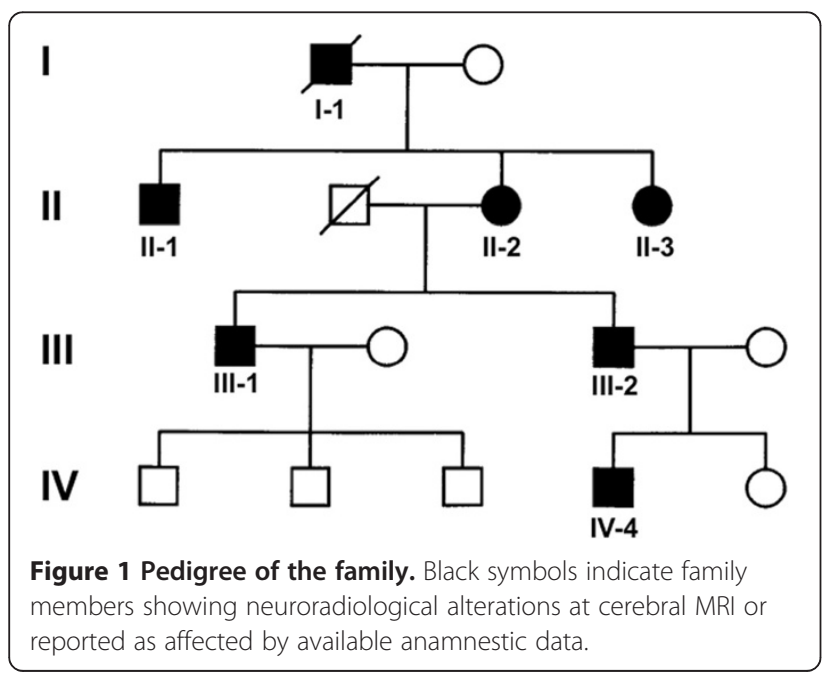

previously symptomatic lesion. Cerebral MRI showed additional widespread lesions: bilateral parietal, bilateral frontal, left temporal and left occipital lobes (Figure 2G-I). Neurological examination showed right facial weakness, absent corneal reflex and mild gait instability.

Patient's mother (II-2) is a 74-year-old woman who was incidentally diagnosed in her sixties with multiple infra- and supratentorial CCMs on cerebral MRI after a transient global amnesia. Cavernous malformations were observed at ponto-mesencephalic junction, left cerebellum, bilateral temporal, right parietal and left frontal cortices (Figure 2A-C). Spinal MRI was normal, but multiple hepatic hemangiomas were found. Last neurological examination (performed in May 2013) was unremarkable. Her father (I-1) died of cerebral haemorrhage when he was in his sixties. Both her brother (II-1) and sister (II-2), aged 78 and 68 respectively, were found to have multiple CCMs, the former experiencing seizures. MRI scan in the proband's 15-year-old nephew (IV-4) also disclosed multiple infra- and supratentorial cavernous malformations (Figure 2J-L). Conversely, spinal MRI was negative.

\section{Molecular studies}

Investigations were carried out according to the guidelines of the Ethical Committee of Istituto Di Ricovero e Cura a Carattere Scientifico Foundation Ca' Granda Ospedale Maggiore Policlinico in Milan and in agreement with Italian and European Union laws.

After obtaining written informed consent, genomic DNA was extracted from peripheral blood lymphocytes of proband (III-1) and two affected relatives (II-2 and III-2).

CCM1/KRIT1 (NM_194454.1), CCM2 (NM_031443.3) and PDCD10/CCM3 (NM_007217.3) coding exons and their intronic boundaries were PCR-amplified using primers and conditions available on request. PCR products underwent direct sequencing using the BigDye Terminator 3.1 protocol on a 3130 Genetic Analyzer (Applied Biosystems). A Restriction Fragment Length Polymorphism assay (PCR-RFLP) was established to detect c.263-10A > G, using restriction endonuclease DraI, which cuts wild-type DNA molecules.

Total RNA was obtained from peripheral blood of the probands (Nucleospin RNA Blood Midi Kit, Macherey Nagel) and retrotranscribed (HiFi Transcriptor cDNA synthesis kit, Roche). Complementary DNA (cDNA) was amplified by PCR using primers encompassing exons 5 and 7. RT-PCR amplicons were subcloned into TOPO-TA vector (Invitrogen) and $1 \mu \mathrm{l}$ of the ligation product was transformed into the XL-10 Gold E. Coli Competent Cells (Stratagene). After overnight growth at $37^{\circ} \mathrm{C}$ on ampicillin plates, twenty colonies were picked and directly sequenced as described above. 


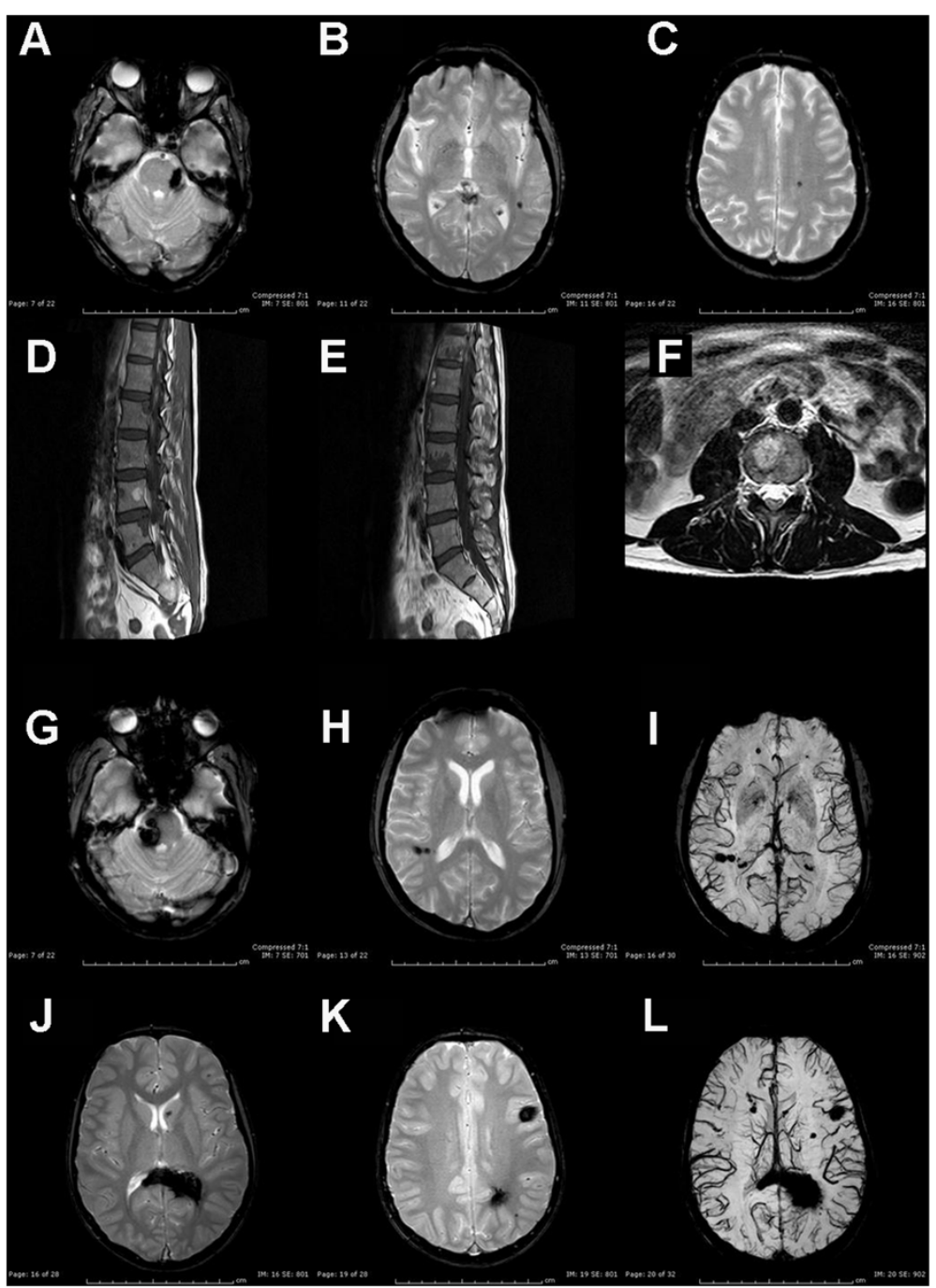

Figure 2 MRI findings. Cerebral MRI in subject II-2 (A-C), III-2 (G-I) and IV-4 and spinal MRI in subject III-1 (D-F). (A-C): axial T2-ffe showing left ponto-mesencephalic angioma (A) and multiple supratentorial cavernous angiomas (left temporal and posterior frontal). (D-F): sagittal T1W-TSE (D-E) and sagittal T1W-TSE (F) showing multiple vertebral hemangiomas D12, L2-L5. (G-I): axial T2-ffe showing right pontine (G) and parietal (H) angiomas. Axial SWI (I) with abnormal right parietal and bilateral frontal venous malformations. (J-L) axial T2-ffe (J-K): multiple bilateral cerebral angiomas with involvement of the splenium of the corpus callosum, periventricular parietal white matter, head of the caudate nucleus, bilaterally, left cortical and subcortical white matter.

\section{Results}

Sequence analysis of CCM2 and CCM3 did not disclose pathogenetic variants. Conversely, a novel nucleotide substitution (c.263-10A > G) was found within intron 5 of CCM1/KRIT1 (Figure 3A). The variant was not observed in 340 ethnic-matched control chromosomes as well as in the following public available databases: CCM mutation database (http://www.angiomaalliance.org/pages.aspx?content=345\&id=289), NCBI SNP (http://www.ncbi.nlm.nih. gov/snp/) and the National Heart, Lung, and Blood Institute (NHLBI) Exome Sequencing Project Exome Variant Server (http://evs.gs.washington.edu/EVS/). PCR-RFLP analysis confirmed the presence of the mutation in proband's mother and an affected brother, supporting the segregation of the defect with the phenotype (Figure 3B). DNA samples from other members of the family were not available for molecular analysis.

The effects of the novel variant were evaluated using Spliceview (http://bioinfo4.itb.cnr.it/ webgene/wwwspliceview_ex.html), Spliceport (http://spliceport.cbcb.umd.edu), ASSP (http://wangcomputing.com/assp/index.html) and Human Splicing Finder (http://www.umd.be/HSF/). The results showed that c.263-10A $>$ G may create a novel acceptor site for exon 6, likely altering the physiological splicing of CCM1/KRIT1 transcript (data not shown). To address this point, we investigated the cDNA retrotranscribed from blood-extracted RNA of the proband and his mother. Sequence analysis detected the inclusion of a 


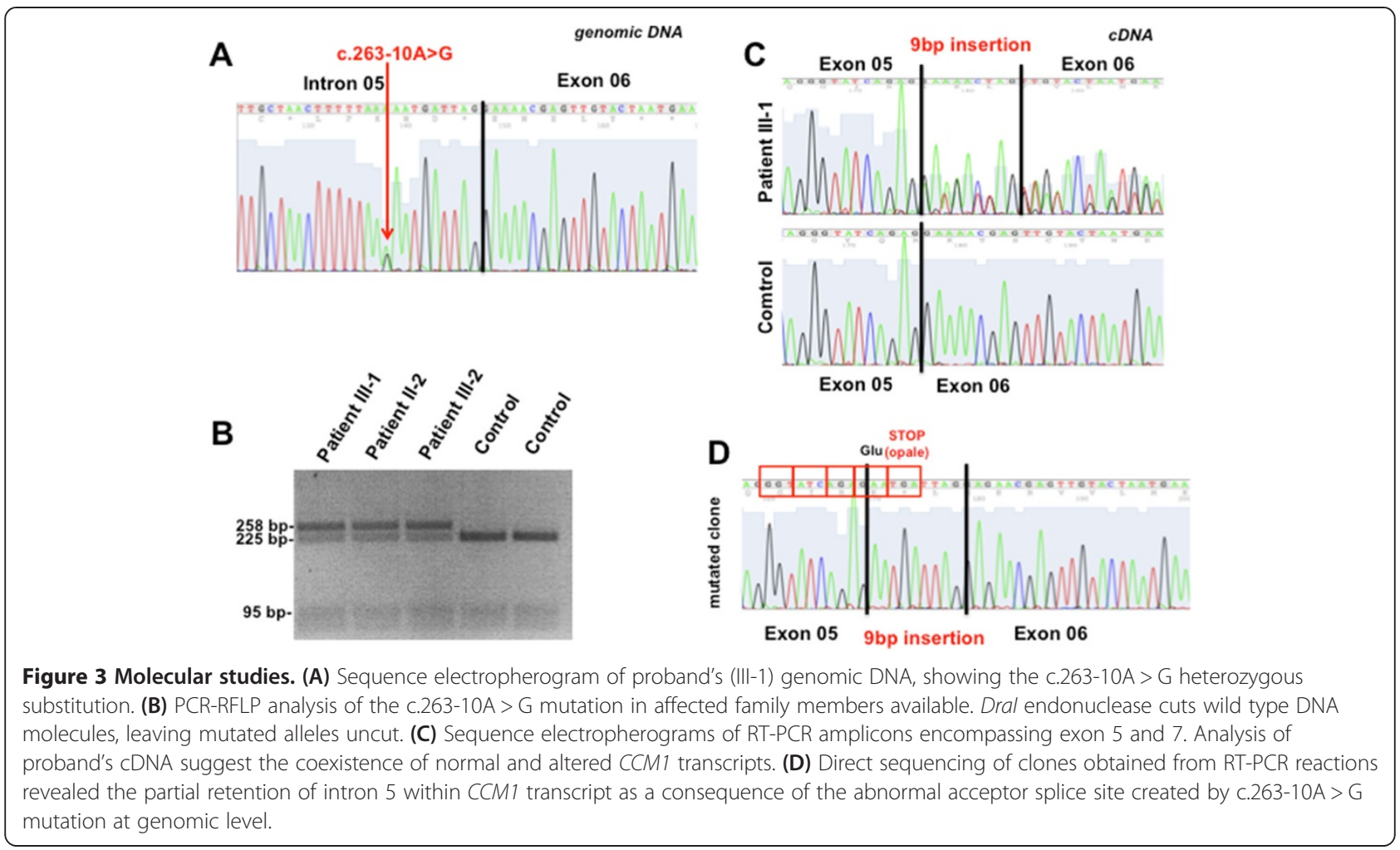

short sequence (AATGATTAG) within patients's cDNA (Figure $3 \mathrm{C}$ ). This finding was confirmed by sequencing clones obtained from RT-PCR fragments encompassing exons 5 and 7 (Figure 2D). The inclusion of the AAT GATTAG sequence is expected to change CCM1 reading frame, resulting in a premature stop codon (p.88insGlufsX1, NP_919436.1).

\section{Conclusions}

The identification of a novel CCM1 mutation in this study expands the number of molecular defects underlining familial CCM. Several elements supports the pathogenicity of c.263-10A > G variant: i) it segregates with the disease in our pedigree; ii) it is absent in a large number of Italian control samples; iii) it affects a nucleotide within a highly conserved region in proximity to intron-exon junction and its substitution is predicted to create an abnormal acceptor splice site; iv) transcript analysis disclosed the inclusion of a short sequence likely altering KRIT1 reading frame and leading to a premature stop codon.

Considering the genetic forms of cerebral cavernous malformations, signs and symptoms involving CNS are more prominent and heterogeneous [8]. Symptomatic patients may present with recurrent headache, seizure, brain haemorrhage or focal neurological deficits $[9,10]$. Clinical findings in our probands are similar to previous reports about CCM1-mutated patients [11].
Few reports have described extra-neural presentations in CCM1-mutated subjects, mainly affecting skin and eye $[12,13]$. Spinal MRI was performed in three members of our family to check for the presence of spinal angiomas, a not unusual finding in familial CCM that may potentially generate complications. Radiological studies only disclosed vertebral hemangiomas as isolated extra-neural disease manifestation in the proband. Despite clinically silent, the number and size of these alterations were unexpected considering patient's age. Further scans confirmed that they were stable over time. Although vertebral hemangiomas are relatively frequent in the general population [14], with an estimated incidence reanging from 10 to $12 \%$, the co-occurrence of vertebral, cerebral and spinal malformations is rare, and it was reported only in two families. The most common clinical symptoms in patients with evidence of vertebral disease were enuresis, back pain, paresthesia and radicular pain due to pathological vertebral fracture [15-17]. This finding was not extended to other members of the family, but hepatic hemangiomas were observed in proband's mother, confirming the association of extra neural hemangiomas and cerebral cavernous malformations due to KRIT1 mutations. A better knowledge of the disease-associated phenotype may lead to an early diagnosis and to an appropriate clinical surveillance in affected patients. 


\section{Consent}

Written informed consent was obtained from the proband, his brother and their mother for publication of this Case report and any accompanying images. A copy of the written consent is available for review by the Editor of this journal.

\section{Competing interests}

The authors declare that they have no competing interests.

\section{Authors' contributions}

Clinical workout: SL, NA, PB. Neuroradiological studies: VC. Molecular Studies: DR. Drafting of manuscript: SL, DR, SC. Critical revision of the manuscript: NB, GPC. All authors read and approved the final manuscript.

\section{Acknowledgements}

Gratitude has to be expressed to the patient for participating in this research. We wish to thank especially the 'Associazione Amici del Centro Dino Ferrari' for their support.

\section{Author details}

'Neurology Unit, IRCCS Foundation Ca' Granda Ospedale Maggiore Policlinico, Milan, Italy. ${ }^{2}$ Dino Ferrari Center, Neuroscience Section, Department of Pathophysiology and Transplantation (DEPT), IRCCS Foundation Ca' Granda Ospedale Maggiore Policlinico, University of Milan, Via Francesco Sforza 35, 20122 Milan, Italy. ${ }^{3}$ Neuroradiology and Interventional Neuroradiology Unit, IRCCS Foundation Ca' Granda Ospedale Maggiore Policlinico, Milan, Italy.

Received: 18 March 2014 Accepted: 28 July 2014

Published: 3 August 2014

\section{References}

1. Rigamonti D, Drayer BP, Johnson PC, Hadley MN, Zabramski J, Spetzler RF: The MRI appearance of cavernous malformations (angiomas). J Neurosurg 1987, 67:518-524

2. Otten P, Pizzolato GP, Rilliet B, Berney J: 131 cases of cavernous angioma (cavernomas) of the CNS, discovered by retrospective analysis of 24,535 autopsies. Neurochirurgie 1989, 35:82-83. 128-131.

3. Labauge P, Denier C, Bergametti F, Tournier-Lasserve E: Genetics of cavernous angiomas. Lancet Neurol 2007, 6:237-244.

4. Rigamonti D, Hadley MN, Drayer BP, Johnson PC, Hoenig-Rigamonti K, Knight JT, Spetzler RF: Cerebral cavernous malformations. Incidence and familial occurence. N Engl J Med 1988, 319:343-347.

5. Pagenstecher A, Stahl S, Sure U, Felbor U: A two-hit mechanism causes cerebral cavernous malformation: complete inactivation of CCM1, CCM2 or CCM3 in affected endothelial cells. Hum Mol Genet 2009, 18:911-918.

6. Sahoo T, Johnson EW, Thomas JW, Kuehl PM, Jones TL, Dokken CG, Touchman JW, Gallione CJ, Lee-Lin SQ, Kosofsky B, Kurth JH, Louis DN, Mettler G, Morrison L, Gil-Nagel A, Rich SS, Zabramski JM, Boguski MS, Green ED, Marchuk DA: Mutations in the gene encoding KRIT1, a Krev-1/rap1a binding protein, cause cerebral cavernous malformations (CCM1). Hum Mol Genet 1999, 8:2325-2333.

7. Fischer A, Zalvide J, Faurobert E, Albiges-Rizo C, Tournier-Lasserve E: Cerebral cavernous malformations: from CCM genes to endothelial cell homeostasis. Trends Mol Med 2013, 19:302-308.

8. Gault J, Sain S, Hu LJ, Awad IA: Spectrum of genotype and clinical manifestations in cerebral cavernous malformations. Neurosurgeny 2006, 59:1278-1284

9. Batra S, Lin D, Recinos PF, Zhang J, Rigamonti D: Cavernous malformations; natural history, diagnosis and treatment. Nat Rev Neurol 2001, 5:659-670.

10. Gross BA, Lin N, Du R, Day AL: The natural history of intracranial cavernous malformations. Neurosurg Focus 2011, 30:E24.

11. Denier C, Labauge P, Brunereau L, Cavé-Riant F, Marchelli F, Arnoult M, Cecillon M, Maciazek J, Joutel A, Tournier-Lasserve E: Clinical features of cerebral cavernous malformations patients with KRIT1 mutations. Ann Neurol 2004, 55:213-220.

12. Grippaudo FR, Piane M, Amoroso M, Longo B, Penco S, Chessa L, Giubettini $M$, Santanelli F: Cutaneous venous malformations related to KRIT1 mutation: case report and literature review. J Mol Neurosci 2013, 51:442-445.

13. Sirvente J, Enjolras $\mathrm{O}$, Wassef $\mathrm{M}$, Tournier-Lasserve E, Labauge P: Frequency and phenotypes of cutaneous vascular malformations in a consecutive series of 417 patients with familial cerebral cavernous malformations. J Eur Acad Dermatol Venereol 2009, 23:1066-1072.

14. Acosta FL Jr, Sanai N, Chi JH, Dowd CF, Chin C, Tihan T, Chou D, Weinstein PR, Ames CP: Comprehensive management of symptomatic and aggressive vertebral hemangiomas. Neurosurg Clin N Am 2008, 19:17-29.

15. Toldo I, Drigo P, Mammi I, Marini V, Carollo C: Vertebral and spinal cavernous angiomas associated with familial cerebral cavernous malformation. Surg Neurol 2009, 71:167-171.

16. Clatterbuck RE, Cohen B, Gailloud P, Murphy K, Rigamonti D: Vertebral hemangiomas associated with familial cerebral cavernous malformation: segmental disease expression. Case report. J Neurosurg 2002, 97:227-230

17. Ropper AE, Cahill KS, Hanna JW, McCarthy EF, Gokaslan ZL, Chi JH: Primary vertebral tumors: a review of epidemiologic, histological, and imaging findings, part I: benign tumors. Neurosurgery 2011, 69:1171-1180.

doi:10.1186/s12883-014-0158-3

Cite this article as: Lanfranconi et al: A novel CCM1 mutation associated with multiple cerebral and vertebral cavernous malformations. BMC Neurology 2014 14:158.

\section{Submit your next manuscript to BioMed Central and take full advantage of:}

- Convenient online submission

- Thorough peer review

- No space constraints or color figure charges

- Immediate publication on acceptance

- Inclusion in PubMed, CAS, Scopus and Google Scholar

- Research which is freely available for redistribution 